\title{
Growth layer and fibre orientation around knots in Norway spruce: a laboratory investigation
}

\author{
Min $\mathrm{Hu}^{1}$ (D) Andreas Briggert ${ }^{1} \cdot$ Anders Olsson $^{1} \cdot$ \\ Marie Johansson ${ }^{1} \cdot$ Jan Oscarsson $^{1} \cdot$ Harald Säll $^{2}$
}

\begin{abstract}
The strength of structural timber largely depends on the occurrence of knots and on the local material directions in the surroundings of such knots. There is, however, a lack of methods for establishing a full dataset of the local material directions. The present research aims at the development and application of a laboratory method to assess the geometry of growth layers and the orientation of fibres in a high-resolution 3D grid within wood specimens containing knots. The laboratory method was based on optical flatbed scanning and laser scanning, the former resulting in surface images and the latter, utilizing the tracheid effect, resulting in in-plane fibre angles determined in high-resolution grids on scanned surfaces. A rectangular solid wood specimen containing a single knot was cut from a tree in such a way that it could be assumed that a plane of symmetry existed in the specimen. By splitting the specimen through this plane through the centre line of the knot, two new specimens with assumed identical but mirrored properties were achieved. On one of the new specimens, the longitudinal-radial plane was subsequently scanned, and the longitudinal-tangential plane was scanned on the other. Then, by repeatedly planing off material on both specimens followed by scanning of the new surfaces that gradually appeared, 3D coordinate positions along different growth layers and 3D orientation of fibres in a 3D grid were obtained. Comparisons between detected fibre orientation and growth layer geometry were used for the assessment of the accuracy obtained regarding 3D fibre orientation. It was shown that the suggested method is well suited to capture growth layer surfaces and that it provides reliable information on 3D fibre orientation close to knots. Such knowledge is of great importance for understanding the properties of timber including
\end{abstract}

Min $\mathrm{Hu}$

min.hu@lnu.se

1 Department of Building Technology, Linnaeus University, Växjö, Sweden

2 Department of Forestry and Wood Technology, Linnaeus University, Växjö, Sweden 
knots. The quantitative data obtained are also useful for calibration of model parameters of general models on fibre orientation close to knots.

\section{Introduction}

\section{Background}

One of the largest advantages of using wood as a building material is that it contributes to global sustainability. Wooden buildings are characterized by low life cycle energy consumption and limited carbon emissions (e.g. Dodoo et al. 2009, 2012), compared to buildings constructed from other commonly used structural materials. Further advantages of using wood are that this material is very strong and stiff in relation to its weight and that it is easy to process. However, the application of wood as building material also means certain challenges. Wood and timber have complicated inner structure and wood material properties vary within a stem of a tree, between boards and even within a single board. One of the most important properties determining strength, stiffness and shape stability of sawn timber is the fibre direction within it. For the strength of boards, the occurrence of knots, together with the local fibre orientation and the local growth layer geometry in the surrounding of knots, is of utmost importance. Knowledge of the local material directions is a prerequisite for development of accurate mechanical models of timber for prediction of strength and eventually for future development of more accurate machine strength grading methods than those applied on the market today. Therefore, there is a need for the development of assessment methods with which accurate determination of local material directions can be achieved.

\section{Growth of clear wood}

Studies on wood formation (e.g. Panshin and Zeeuw 1980; Plomion et al. 2001) show that wood cell division takes place in the cambium zone of the tree. This zone is situated between xylem (the wood) and phloem (the bark). The cambium cell is characterized by the ability to divide itself into both xylem and phloem cells depending on the direction towards which the cambium cell divides. A growth layer is herein defined as a xylem cell layer that is developed at a certain point of time in the cambium area of the tree. Studies on wood anatomy show that wood fibres tend to grow in a spiral pattern around the trunk of the tree. Such growth is known as spiral grain, and previous research has shown that the spiral grain angle within clear wood varies with the distance from the pith (Harris 1988) and that the average value for Norway spruce (Picea abies (L.) H. Karst) is normally below $3^{\circ}$ (Säll 2002).

\section{Hypothesis and models for fibre orientation around knots}

A model on how fibres integrate a branch with the trunk was presented by Shigo (1985), who hypothesized that branch tissue begins to develop before trunk tissue 
early in a growth season. In this context, the concept of tissue refers to a group of fibres of similar makeup. The branch xylem encircles the branch base and forms a collar in which the tissue meets on the trunk below the branch, see Fig. 1. This branch collar is enveloped later in the growth season by the trunk tissue in a sense that the trunk tissue holds the branch collar firmly in place. Thus, the branch is, as shown in Fig. 1, structurally attached to the trunk by a series of trunk collars that envelop the branch collars every growth season. Shigo's hypothesis is based on large samples of dissections through branches of trees, debarking of branch junctions at varying time of a particular growth season, conduction tests using dye and microscopic observations etc. Shigo $(1985,1986)$ has often been cited, when branch attachment to trunks was discussed, but his explanation has also been questioned. For example, Nelly (1991) studied the hydraulic architecture, i.e. how sap flows at the branch junction, by performing conduction tests using dyes. In this study, water-soluble dye was injected into the stem through predrilled holes beneath branches of trees at various times over a growth season. Thereafter, stems and branches were locally peeled and the uncovered surfaces were examined to trace the pattern of dye translocation. It was shown that patterns of dye movement did not vary with time of the year and thus the experiment did not support Shigo's hypothesis on the varying patterns of branch and trunk tissues over each growth season. In the study, however, the predrilled holes for the dye injection were $1 \mathrm{~mm}$ in diameter and $5 \mathrm{~mm}$ deep. Dye in such holes could penetrate both the branch collar and the trunk collar described in Shigo's model (Fig. 1) making it hard to observe the varying patterns of branch and trunk tissues over a growth season. Moreover, conduction tests with dyes, both by Shigo (1985) and by Nelly (1991),

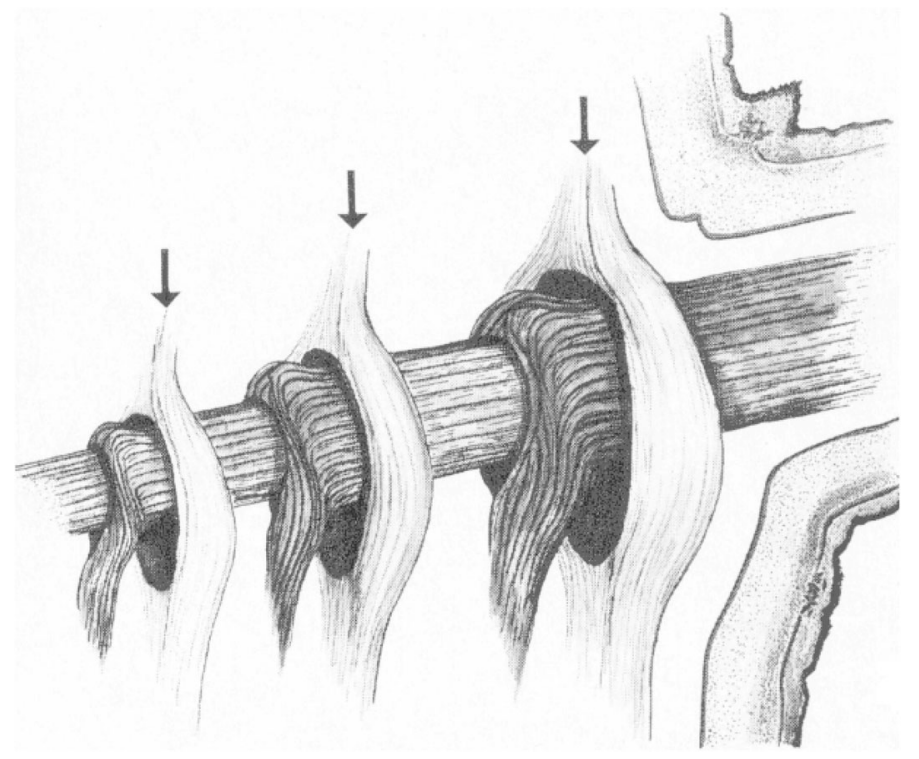

Fig. 1 Illustration of Shigo's branch attachment theory (originating from Shigo (1985) and reprinted with permission of Shigo and Trees, Associates LLC) 
confirmed that sap flows to the branches from below the branch and not from above. Therefore, Shigo concluded that 'the trunk tissue from above the branch does not connect with the branch' (Shigo 1986). Slater and Harbinson (2010) argued, however, that branch and stem may be connected by, for example sclereids tissue. Such tissue plays no significant role in water transport, and therefore, it would not conduct dye, but it provides mechanical support.

Attempts to describe the fibre course in the vicinity of knots have been made at least since the 1970s. Goodman and Bodig (1978), who introduced a mathematical expression of generalized grain angle variation occurring in the longitudinaltangential plane in the surrounding of knots, suggested a 'flow-grain' analogy. According to this analogy, the fibre deviation in the vicinity of knots can be modelled by the potential streamlines of a fluid passing around an elliptically shaped obstacle. The concept of flow-grain analogy has been motivated by the visual similarity between the mentioned streamlines and the actual grain pattern seen on longitudinal-tangential surfaces. Regarding this similarity Goodman and Bodig (1980) gave an explanation that relates to the function of the living tree. They claimed that an actual laminar flow of fluids exists around the knot at the time when the cells are produced, since the main fluid transportation occurs in the growing cells. This two-dimensional flow-grain analogy was adopted and extended by Foley (2001) to a three-dimensional model describing the geometry of knots and related fibre distortion. An important attribute of this paradigm is a mathematical expression to capture the geometry of growth layers in the vicinity of knots by which the fibre angle in the longitudinal-radial plane is determined. Models of timber in which the paradigm/model suggested by Foley was integrated have also been established and used for detailed structural mechanics analyses (Hackspiel 2010; Lukacevic and Füssl 2014). Another starting point to describe the fibre course around knots is the assumption that the fibres are oriented such that the principal stresses during growth of the tree coincide with the fibre orientation (Mattheck 1997). An iterative procedure including finite element analysis can be applied to determine the fibre orientation on the basis of this assumption. Lang and Kaliske (2013) discussed and compared the models based on principal stresses and the flowgrain analogy, respectively. They showed that for fibre orientation around a round knot both models provide similar results that appeared to be reasonable.

\section{Laser scanning and the tracheid effect}

The fibre orientation in the plane of a softwood surface can be determined by utilizing the so-called tracheid effect. This means that when the surface is illuminated by laser rays, light spreads more along fibres than across (Matthews and Beech 1976; Soest et al. 1993) such that the light spots on the wood surface become elliptical, rather than circular in shape. The major axis of the elliptically shaped light spot thus gives an indication of the fibre orientation in the plane of the surface. Figure 2 shows (a) a wood surface including a knot and an elliptically shaped light spot resulting from illumination by a laser ray in this position, and (b) determined in-plane fibre orientation locally on the wood surface obtained by illuminating different positions of the wood surface, in a grid, by laser rays. The tracheid effect 
Fig. 2 a An elliptically shaped light spot on a wood surface that is illuminated locally by laser rays, and $\mathbf{b}$ determined in-plane fibre direction field on a wood surface

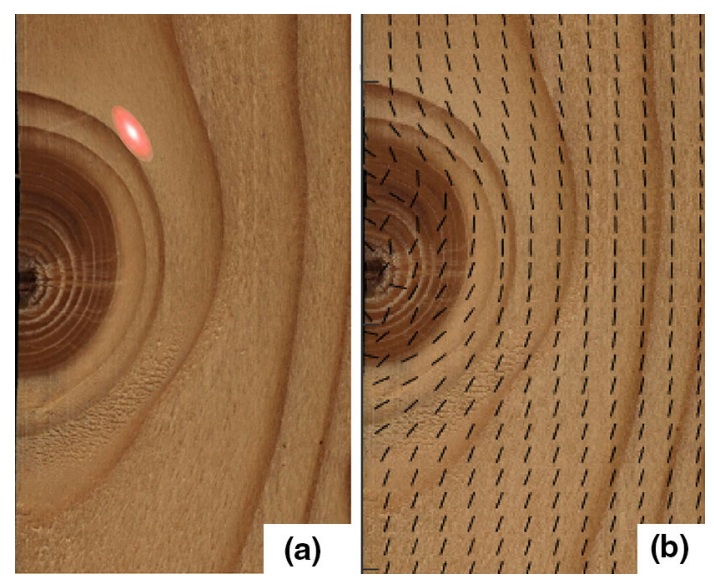

has often been used, also in industrial processes, to determine the in-plane fibre angle on wood surfaces (e.g. Olsson et al. 2013; Olsson and Oscarsson 2017).

\section{Objectives and assumptions}

The fibre orientation models discussed above, i.e. the flow-grain analogy, the principal stress model and the three-dimensional model presented by Foley (2001), all give plausible results. Qualitatively they seem to comply well with the actual fibre orientation in the vicinity of knots. However, there is a lack of detailed experimental data with which such models can be assessed quantitatively and further developed. Therefore, the present research aims at (1) the development of a laboratory method to determine 3D growth layer geometry, (2) the development of a laboratory method to determine high-resolution 3D fibre orientation in the vicinity of knots, and (3) local determination of longitudinal, radial and tangential material directions, in any position within an investigated wood specimen. A further aim is to assess the obtained accuracy of determined material directions.

An assumption was made that a plane of symmetry exists through the knot centred within an examined specimen, which means that the knot itself, the growth layers and the fibre orientation are symmetric with respect to this plane (Hu et al. 2016). This also means that the influence of spiral grain on fibre distortion related to knots is ignored. The work aiming at determination of the growth layers is based on tracing a growth layer in images obtained by using a flatbed scanner. The identification of the fibre orientation is based on laser scanning and utilization of the tracheid effect.

\section{Material and specimen preparation}

The study was carried out on Norway spruce (Picea abies (L.) H. Karst). Two trees were selected from two stands at the locality of Kråkenäsryd Gårdsby $\left(56^{\circ} 57^{\prime} 17^{\prime \prime} \mathrm{N}\right.$, $\left.14^{\circ} 54^{\prime} 06^{\prime \prime} \mathrm{E}\right)$ in southern Sweden. These stands are well documented. During the 
years, 1953 and 1962, a series of pre-commercial thinning experiments were established in Sweden. They involved in total 28 localities, which were divided into 140 stands. The locality and stands at which the two trees had been felled were part of the experiments. The trees used in this study were planted in the spring of 1962 with 4-year-old trees of the provenience Nödebo F71, Denmark. The locality of Kråkenäsryd Gårdsby consisted of nine stands and the purpose was to study volume and quality of timber after thinning to different numbers of remaining stems. The two trees used in this study were selected from two stands which after the precommercial thinning had 600 and 1800 stem/hectare, respectively.

In total, 12 specimens with the shape of rectangular solids were manufactured from the two trees. Illustration of one such specimen is shown in Fig. 3c-e. Each specimen contained one centred knot with substantial margins of wood in longitudinal and tangential direction and the size in the radial direction, i.e. the zdirection as shown in Fig. 3c, was at least $100 \mathrm{~mm}$. Furthermore, the following characteristics were considered when selecting the trees and preparing the

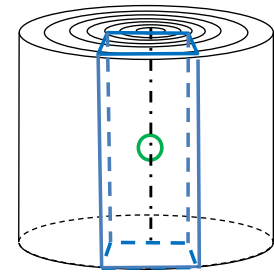

(a)

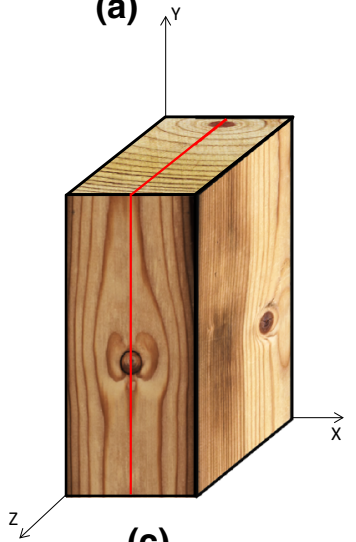

(c)

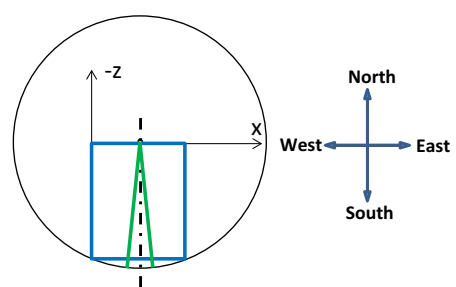

(b)

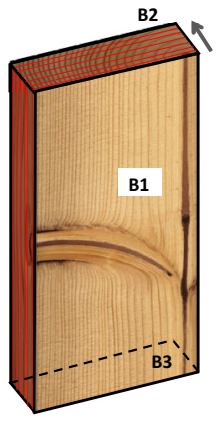

(d)

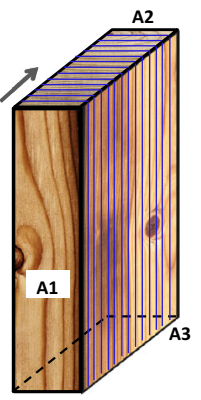

(e)

Fig. 3 Illustration of specimen preparation. a A potential specimen is indicated by blue lines within a $\log$; the green circle indicating the position of a knot and the dot-dashed black line the assumed plane of symmetry. b The potential specimen in $x z$-view and a sketch showing the four cardinal points. c An example of an obtained specimen shaped as a rectangular solid containing a centred knot. The specimen was split through the plane of symmetry, i.e. along the red lines in (c) into two parts (d) and (e) that were planed, in steps as indicated by the red lines in (d) and blue lines in (e). A global coordinated system $(x y z)$, shown in (c), was defined with coordinate axes parallel with the edges of the specimen. The arrows in (d) and (e) show the direction and sequences of the planing of the two parts. The surfaces marked with capital letters and numbers (e.g. B1 and A1) are the object surfaces in the scanning process 
specimens: the annual ring width should vary from narrow to wide between specimens; the knots within the specimens should have minimal disturbances from other branches/knots; the knot should preferably represent cases of different angles between the knot and the pith of the tree and even different geometries of the knot itself. To avoid cracking, the specimens were slowly kiln dried in a laboratory kiln to an average moisture content of about $18 \%$.

In the study presented in this paper, only one specimen was examined. This specimen was cut from the stem grown at the site with 1800 stem/hectare. In the tree, the knot contained in the specimen was located $6.1 \mathrm{~m}$ above the ground and directed southward (Fig. 3b). The specimen had, referring to the global coordinate system defined in Fig. 3c, a dimension of $95(x) \times 559(y) \times 105(z) \mathrm{mm}^{3}$. The pith of the tree from which the specimen had been cut was positioned within the specimen close to the $x y$-plane. The specimen had an average density of $418 \mathrm{~kg} / \mathrm{m}^{3}$ at about $18 \% \mathrm{MC}$, and it included about 29 annual rings with an average width of $3.5 \mathrm{~mm}$. The main knot in the specimen was a live knot up until about annual ring number 13 and was thereafter included in the stem of the tree as a dead knot. The specimen was split through an assumed plane of symmetry as indicated by the red line in Fig. 3c.

\section{Method}

The assumed plane of symmetry, as discussed above, was a longitudinal-radial plane, which contained the pith of the knot as well as the pith of the log, see Fig. 3d. After splitting of the specimen, the laboratory part of the work was a process alternating between scanning and planing of the two parts. The part of the specimen in which the half knot is at the left edge is herein called part A (Fig. 3e), whereas the other part is called part B (Fig. 3d). The two parts were scanned and planed differently, as described below. It should be noted that flatbed image scanning, described in "Flatbed image scanning and determination of growth layer geometry" section, was carried out between each planing, whereas the laser scanning, described in "Laser scanning and determination of fibre orientation in 3D" section, was in most cases performed for every second planing.

\section{Flatbed image scanning and determination of growth layer geometry}

The flatbed image scanning was intended for the determination of growth layer geometry. In practice, positions belonging to a growth layer, which for example can be identified by the colour change at the interface between latewood and earlywood, can be recognized in an image of the wood surface. Thus, a growth layer within a specimen can be determined by examining a series of images of the surfaces through the planing sequence indicated by the arrows in Fig. 3d, e, giving planes with different position in $x$ - and $z$-direction, respectively.

A flatbed scanner of make Plustek OpticBook A300 (from Plustek Technology Inc, Santa Fe Springs, California), see Fig. 4a, was used to obtain the surface images of the specimen. The scanner uses a 4-line colour (Red, Green, Blue and Grey) 
charge-coupled device (CCD) image sensor; the output image used in this study was in TIF format and had a resolution of $600 \mathrm{dpi}$ (dots/pixel per inch).

For part A, surfaces A1-A3 were first scanned. Thereafter, surface A1 was planed off $1 \mathrm{~mm}$ in (negative) $z$-direction. The new surface A1 that appeared after the planing and the corresponding A2-A3 surfaces were then scanned again. The same procedure was repeated until the size of the remaining specimen was too small to be planed off any further. Flatbed scanning was also performed on part B but with a different arrangement. Similarly, surfaces B1-B3 were first scanned, then surface B1 was planed off to remove material of $1 \mathrm{~mm}$, but in this case the material was removed in (negative) $x$-direction. The new surface B1 and the corresponding surfaces B2-B3 were then scanned again. The same scanning and planing procedure was also repeated until the size of the remaining part was too small to be planed off any further.

A key concern of the flatbed scanning was to ensure that the scanned surfaces A1 and B1 were well defined in relation to a global coordinate system defined for the original specimen, i.e. before splitting into two parts. This was done by making use of reference points and lines. Before the splitting, 16 reference points related to the global coordinate system were marked on the specimen. These points are shown and numbered in Fig. 4b. After the splitting, two additional points, marked as no. 17 and 18 in Fig. 4c, were applied on surface B1. The reference points were then used to determine the reference lines, which are shown as dashed lines in Fig. 4c, d. These lines formed areas of $47(x) \times 420(y) \mathrm{mm}$ on A1 and $420(y) \times 105(z) \mathrm{mm}$ on B1. The areas were considered sufficiently large to capture the complete fibre course, i.e. the gradual development of fibre orientation in all coordinate directions, due to the knot. Areas slightly larger than these were scanned, and the specimens were carefully placed in the scanner to ensure that the edges of the scanned surface aligned as good as possible with the edges of the scanned image. This made it feasible to crop the image afterwards to the reference lines. In this way, a welldefined area was captured and a reliable mapping from the image system (in pixels) to the global coordinate system (in millimetres) was achieved.

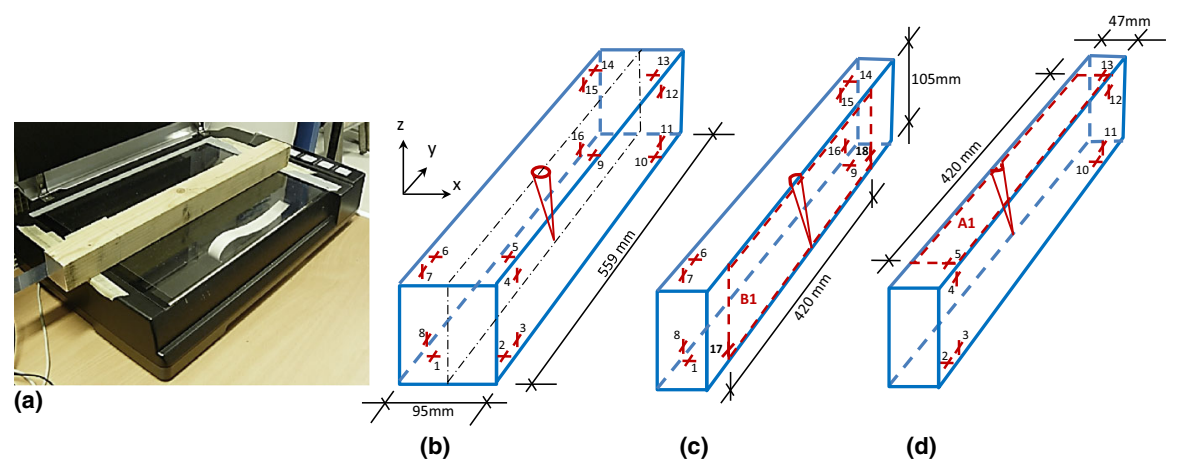

Fig. 4 a Flatbed scanner of make Plustek OpticBook A300, used to obtain images of wood surfaces. b-d Sketches showing reference points, reference lines and flatbed-scanned areas on the two parts obtained after splitting 
From the flatbed scanning, serial images of surface A1 and B1 were obtained. Based on these images, it was possible to manually collect positions, relating to the global coordinate system, belonging to the same selected growth layer. By means of such positions, it was possible to determine 3D geometry of individual growth layers. This was done using the software MATLAB, and the procedure of establishing a growth layer was performed, for example for part $\mathrm{A}$ as follows:

1. Select a growth layer at the interface between latewood and earlywood within an annual ring, for example ring no. 12 from the pith of the log; go through the images of surface A2 to find the range of $z$-position within which the selected annual ring was visible on A1.

2. Load one flatbed image of A1 within the range into MATLAB and visualize it in the figure window; identify the annual ring on the current image and manually sample (of course, this could also be automated) a number of points on the growth layer the $y$-position of which ranged between 0 and 420. An example of such sampled points together with the flatbed image on which the points were selected is shown in Fig. 5.

3. Transform the collected positions from the image (pixel) system to the global coordinate system, to obtain the corresponding 3D coordinates.

4. Repeat steps 2 and 3 for all the images of surface A1 within the range mentioned in step 1. All the points eventually collected represent a data set, which comprises scattered 3D coordinates selected from each image of every $1 \mathrm{~mm}$ along the $z$-direction.

5. Interpolate linearly between the collected points to establish a $3 \mathrm{D}$ grid surface representing the growth layer.

6. To get a normal direction of the growth layer surface, for a certain position on the surface, establish a bi-cubic polynomial that locally gives a least square fit to the surface established in step 5 and use this bi-cubic polynomial surface for calculation of the normal direction.

\section{Laser scanning and determination of fibre orientation in 3D}

The in-plane fibre orientation on the surfaces of the specimen was obtained by laser scanning and utilization of the tracheid effect, resulting in the kind of information as is shown in Fig. 2b. The laser scanning was performed using a self-developed laboratory scanner including a laser source and a camera, see Fig. 6. By means of

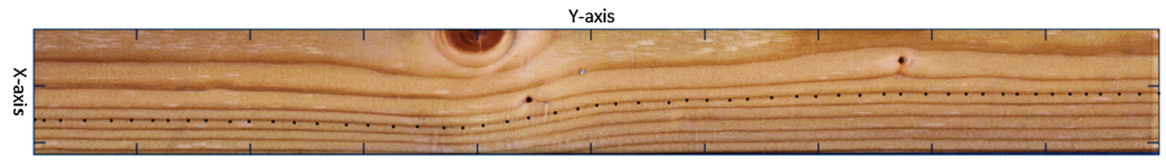

Fig. 5 Example of results of sampled points on a growth layer. The colour image displayed is the flatbed scanning image of surface A1 at $z$-position of $50 \mathrm{~mm}$. The black dots are the manually sampled points from the colour image. The figure is drawn with respect to the image (pixel) system, and the colour image has been cropped to the reference lines 
this scanner, surfaces can be illuminated by a dot laser at one position at a time. Moving the laser and the camera to new positions in a well-defined grid in relation to the piece of wood investigated, photos of laser spots at each grid position can be taken. This results in knowledge of the in-plane fibre orientation over the chosen grid, the latter being equivalent with the in-plane scanning resolution. The in-plane fibre directions shown in Fig. 2b were obtained using the laboratory scanner in the described way. Note that the in-plane fibre orientation field displayed (Fig. 2b) follows the fibre flow around the knot just as can be expected. The indicated fibre directions within the knot area are, however, more or less randomly oriented. This is because the fibre direction here is actually almost perpendicular to the displayed plane. Due to this, the light spots from the dot laser become more or less circular in shape and the small in-plane component of the true 3D fibre direction in positions within the knot cannot be determined with certainty. A more detailed description of the laboratory scanner is given in Briggert et al. (2016).

As described in "Flatbed image scanning and determination of growth layer geometry" section, flatbed scanning was repeatedly carried out on surfaces A1 and B1, see Fig. 3d, e, after material of thickness $1 \mathrm{~mm}$ was planed off between each scanning. This means that the flatbed scanning resolution in the direction of the arrows shown in Fig. 3d, e was $1 \mathrm{~mm}$. Laser scanning using the laboratory laser scanner was also repeatedly carried out on surfaces A1 and B1, but such scanning was not carried out after each planing. The reason for this was that high-resolution scanning using the laboratory scanner is a time-consuming process and a resolution as high as $1 \mathrm{~mm}$ throughout was not necessary. For surface A1, the laser scanning was carried out after every second planing, implying a scanning resolution of $2 \mathrm{~mm}$ in the $z$-direction. For surface B1, the laser scanning resolution varied in negative $x$ direction, i.e. in the direction of the arrow shown in Fig. 3d. In the part of specimen $\mathrm{B}$ that included the knot, laser scanning was performed after each planing, i.e. with a

Fig. 6 Laboratory scanner used to obtain in-plane fibre orientation fields over surfaces of wood specimens

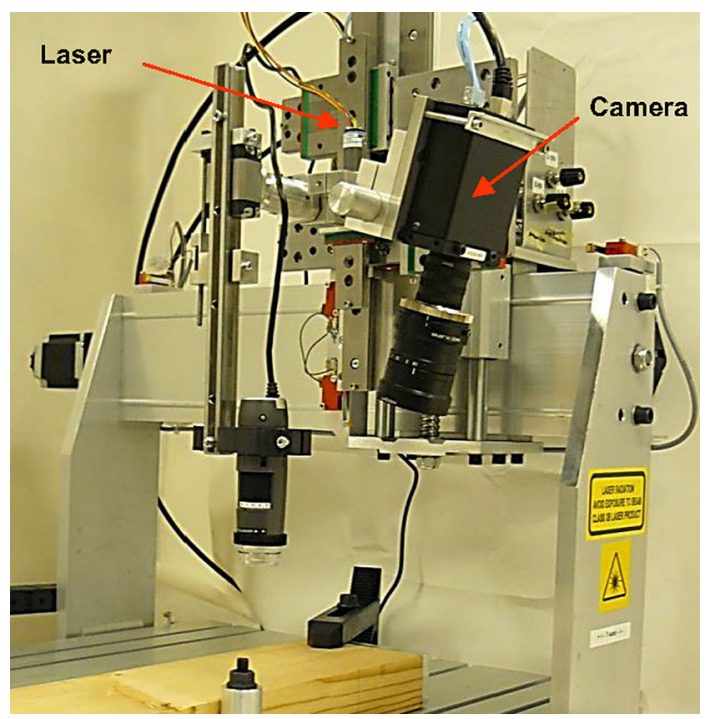


resolution of $1 \mathrm{~mm}$ in negative $x$-direction. Thereafter, the scanning resolution was $2 \mathrm{~mm}$, i.e. scanning after every second planing, for another 20-mm interval in negative $x$-direction. Finally, the resolution was $4 \mathrm{~mm}$, i.e. scanning after every 4 th planing, for the remaining $15 \mathrm{~mm}$ of the specimen, i.e. at a considerable distance from the knot.

Regarding the size of the laser scanned areas and the chosen in-plane resolution, each A1 and B1 surface was scanned over a pre-defined area of $40(x) \times 284(y) \mathrm{mm}^{2}$ and $284(y) \times 96(\mathrm{z}) \mathrm{mm}^{2}$, respectively, in which the knot was centred within the considered range in $y$-direction. Each pre-defined area was divided into two different sub-areas with different scanning resolutions. Inner subareas, covering the knot and its close surroundings, of $40(x) \times 140(y) \mathrm{mm}^{2}$ on A1 and $140(y) \times 96(z) \mathrm{mm}^{2}$ on B1, respectively, was scanned in a $2 \times 2 \mathrm{~mm}^{2}$ grid, whereas the remaining outer parts of the pre-defined areas were scanned in a $4 \times 4 \mathrm{~mm}^{2}$ grid. Through the laser scanning procedure, including successive removal of material that was planed off, followed by scanning of new surfaces that appeared, in-plane fibre directions on surfaces A1 and B1 were obtained in a 3D grid within the investigated specimen.

The assumption regarding symmetry in the specimen shown in Fig. $3 \mathrm{c}$ implied that the fibre direction in a position within part A had the same, or actually mirrored (with respect to the plane of symmetry) direction as the one in the corresponding position in part B. Thus, scanning of both A1 and B1 surfaces means that two different in-plane fibre angles, i.e. one angle in the $x y$-plane and one in the $y z$-plane, could be determined in virtually the same position in the specimen. These two angles, indicated by $\alpha$ (in the $x y$-plane) and $\beta$ (in the $y z$-plane) are shown in Fig. 7. With knowledge of both angles in the same $x y z$-position within the wood specimen, a $3 \mathrm{D}$ vector that represents the fibre orientation in that position was uniquely determined as

$$
v=\left[\begin{array}{lll}
\cos (\alpha), & \sin (\alpha), & \frac{\sin (\alpha)}{\tan (\beta)}
\end{array}\right]
$$

In Fig. 7, a calculated direction vector $v$ is represented by a thick black arrow. Thus, as a result of the described procedure, such direction vectors were achieved in every position of the 3D grid for which in-plane fibre directions were determined within the scanned volume. Of course, the fibre direction in any arbitrary point within the volume can be achieved by performing linear interpolation between the neighbouring direction vectors in the grid.

\section{Verification of the established 3D fibre orientation}

Foley (2003) made the reasonable assumption that fibres are orientated within a growth layer formed during a certain period of time, i.e. within an annual ring or even within a fraction of an annual ring. This assumption was not utilized herein when determining the local fibre direction and thus it can be used for verification, comparing the determined local 3D fibre directions with the orientation of the growth layer surfaces. Following Foley's assumption, accurately determined fibre 
Fig. 7 In-plane fibre angles, $\alpha$ and $\beta$, that uniquely determine a $3 \mathrm{D}$ vector (black arrow) representing the local fibre orientation. The angles $\alpha$ and $\beta$ are obtained from the laser scanning of surfaces A1 and B1, respectively

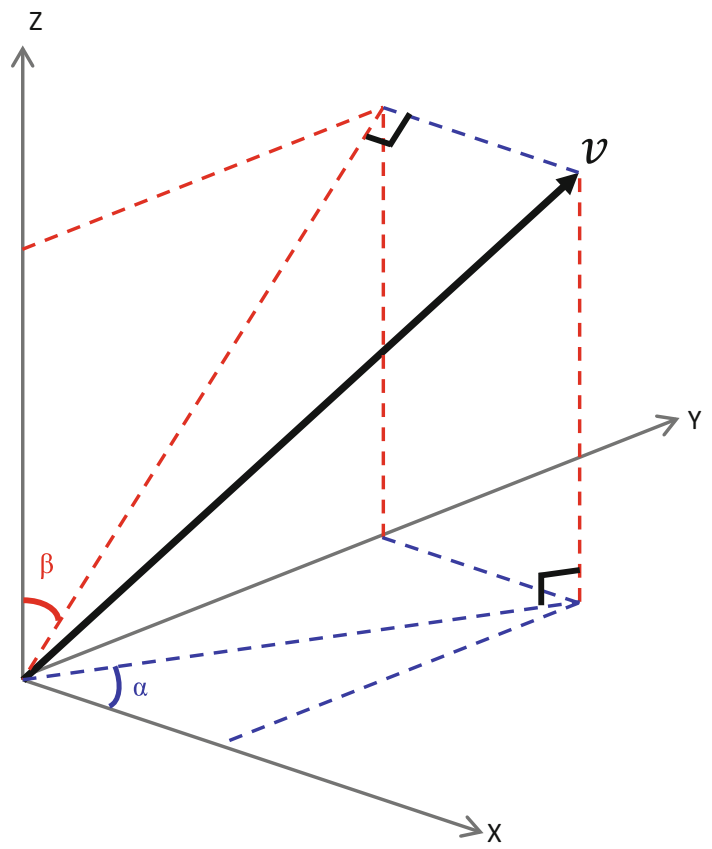

orientation vectors should have a $90^{\circ}$ angle to the normal of the growth layer surface in the same position. It should be noted, however, that any discrepancy, i.e. such angles being different from $90^{\circ}$, may depend on errors of the determined shape/ orientation of the growth layer as well as on errors of the determined fibre directions.

\section{Results and discussion}

In this section, results with respect to the established geometry of growth layers, inplane fibre directions obtained from laser scanning of surfaces and calculated 3D direction vectors representing local fibre orientations are presented. Furthermore, the established 3D vectors are verified and examined by visualizing them in relation to the growth layer surfaces. The results regarding fibre orientation are based on measurements carried out on the split specimen shown in Fig. 3, i.e. on measurements of both part A and part B. However, the 3D growth layer geometry displayed is based solely on scanning of specimen A.

\section{Growth layer geometry}

Two surfaces of growth layers established in the way described in "Flatbed image scanning and determination of growth layer geometry" section are shown in Fig. 8, in which the growth layer surface obtained for part A was symmetrically copied for part B for the purpose of visualization. The black dots on the surfaces in Fig. 8a and 
$\mathrm{b}$ are the manually collected positions from the flatbed images of surface A1. Figure $8 \mathrm{c}$ shows a surface image in which the two growth layers can be identified. The yellow surface is within the dead knot region, whereas the blue surface represents a growth layer within the live knot region. These surfaces correspond with the dashed red and blue lines, respectively, in Fig. 8c. From the results, it is clear that branches/knots have a pronounced local effect on growth layers in the sense that they cause a local swelling around the knot, whereas the bulk of the growth layer form a cylindrically shaped surface, the longitudinal direction of which is close to parallel with the pith of the tree/log.

\section{In-plane fibre direction}

Figure 9 shows in-plane fibre directions, identified on wood surfaces by means of laser scanning. Figure 9a shows the orientation of an A1 surface (blue colour) and a B1 surface (red colour) in relation to the coordinate system employed, along with

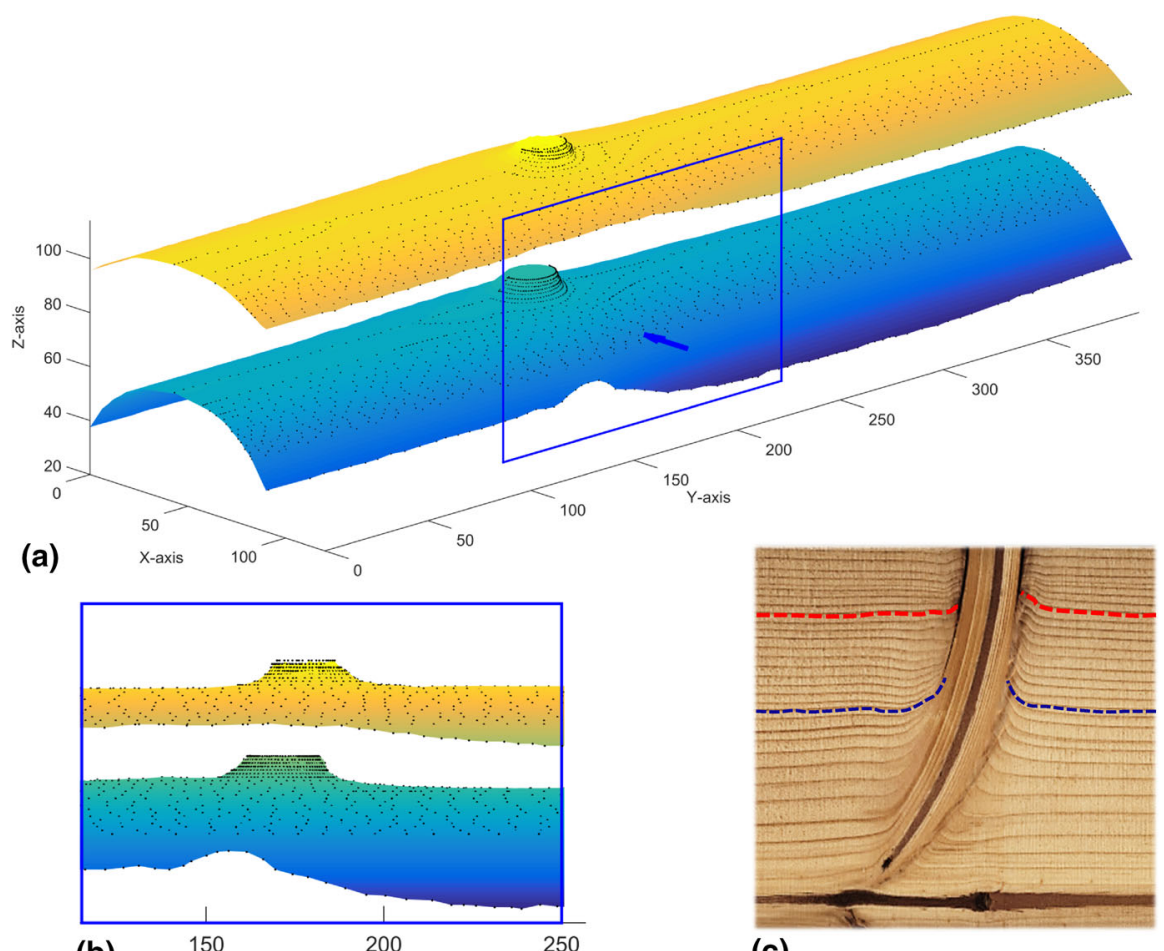

(b) Y-axis

(c)

Fig. 8 a Established surfaces of a growth layer within the live knot region (blue surface) and within the dead knot region (yellow surface). The black dots are the manually collected positions from different images of the repeatedly planed surface A1 (cf. Figs. 3e, 5). b The same growth layer surfaces within the indicated frame in (a) in an $y z$-view. c A photograph of part of the surface B1 through the pith of the knot. The blue dashed line marks the growth layer in the live knot region and the red dashed line marks the growth layer in the dead knot region 
detected in-plane fibre directions of these surfaces. The B1 surface shown is the one coinciding with the assumed plane of symmetry through the investigated specimen (cf. Fig. 3c-e). The areas within the frames exhibited in Fig. 9a, i.e. areas close to the knot, were laser scanned in a 2D grid of $2 \times 2 \mathrm{~mm}^{2}$. In (c-d), the areas framed in (a) are also shown, enlarged, in $y z$-view and in $x y$-view, respectively. (b) shows a photograph of the A1 surface including the area close to the knot, i.e. the same area as represented in (d). Inserted in (c) is a photograph of part of the B1 surface.

Large fibre deviations caused by the knot can be observed on both the A1 surface and the B1 surface (Fig. 9c, d) in the vicinity of the knot, whereas the fibres are more or less parallel with the $y$-direction at positions away from the knot. The identified fibre directions agree well with what is visible on the photographs of the

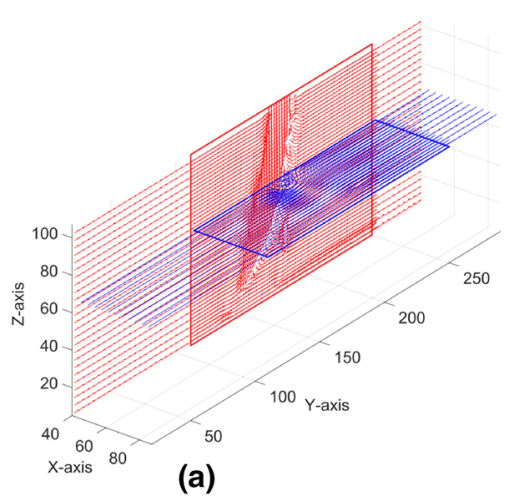

(a)

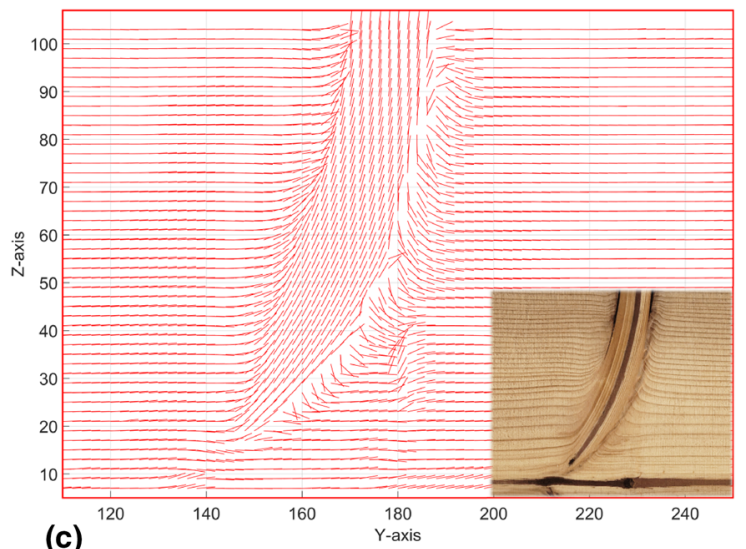

(c)

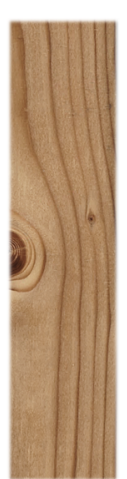

(b)

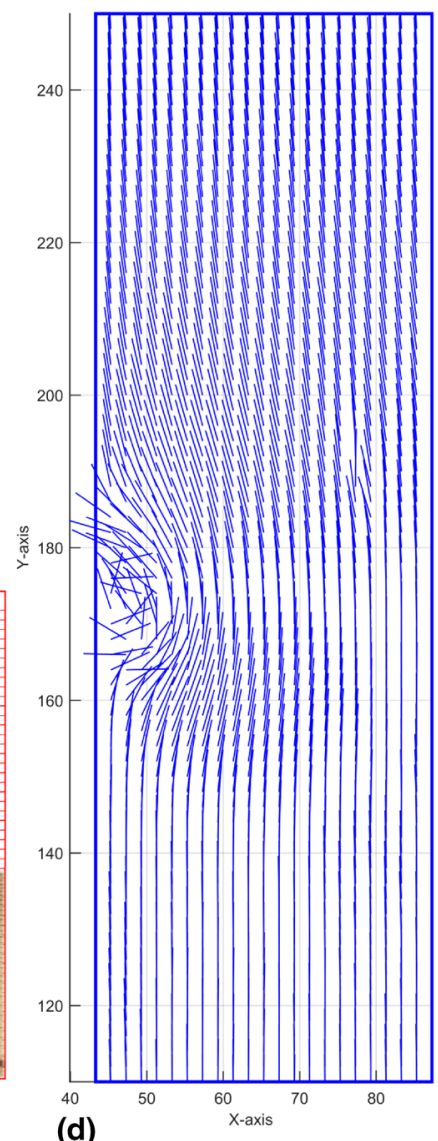

Fig. 9 Identified in-plane fibre orientation from laser scanning of two wood surfaces, A1 and B1 (cf. Fig. 3d, e), along with photographs of the two surfaces. a Orientation of the surfaces in relation to the employed coordinate system, the resolution of the plotted fibre directions is $2 \times 2 \mathrm{~mm}$ within, and $4 \times 4 \mathrm{~mm}$ outside, indicated frames. b Photograph of surface A1 and $\mathbf{c}$ photograph of part of the surface B1 (infolded at the bottom right). c, d Enlargements of the fibre directions within the indicated frames in (a) on surfaces B1 and A1, respectively, in a $y z$-view and an $x y$-view, respectively 
two surfaces. On the $x y$-plane (Fig. 9d) in an area close to the knot, fibres gradually deviate from the $y$-direction and pass around the knot. Inside the knot area, the calculated fibre directions are orientated more or less at random. The latter is due to the fact that the knot fibres are mainly orientated perpendicularly to the displayed plane, resulting in the laser light spots on the surface becoming close to circular, rather than elliptical in shape. In turn, this causes uncertainties in determined major axis directions of the light spots, as explained in "Laser scanning and the tracheid effect" section. The actual fibre orientation within the knot is best shown in the $y z-$ plane (Fig. 9c). Results of the in-plane fibres on the yz-plane (Fig. 9c) also agree well with what can be seen on the infolded photograph here; the fibre direction deviates from the $y$-direction to the knot direction when approaching the knot. Below the knot, but only in the live knot region, the change in fibre orientation is smooth, whereas in the region just above the knot the transition seems to be rather irregular. Considering Shigo's knot formation theory this is not very surprising since according to Shigo the branch integrates with the stem only from below.

\section{Three-dimensional fibre orientation}

In Fig. 10, fibre directions in 3D, established as described in "Laser scanning and determination of fibre orientation in 3D" section, are indicated in positions on one growth layer surface, namely the one that is displayed with blue colour in Fig. 8a, b. The fibre direction vectors are in Fig. 10 represented by small black arrows in positions corresponding to the manually collected positions of this particular growth layer surface (cf. Fig. 5). In most positions on the growth layer surface, the small black arrows that would indicate the fibre orientation are visible. However, in certain positions the arrows are not, or only partly, visible, because the direction of the vector is going 'downwards' in relation to the shown growth layer surface and is thus not visible in the shown view of the surface. In Fig. 10a, b, showing the growth layer surface in 3D view and in $x y$-view, respectively, it can be seen that vectors representing the fibre direction at some distance from the knot flow around it. It also seems like determined fibre directions coincide rather well with the growth layer surface, i.e. that the normal directions of the growth layer surface, locally, is close to $90^{\circ}$ to the black arrows representing the local fibre orientation. In particular, this can be seen in Fig. 10c (showing the growth layer and fibre orientation in yz-view) just below the knot. Here, the black arrows are clearly parallel with the contour of the growth layer.

\section{Assessment of accuracy of obtained results}

As discussed in "Verification of the established 3D fibre orientation" section, an accurately determined fibre direction vector should have a $90^{\circ}$ angle to the normal direction of the growth layer surface in the same position. Any discrepancy from this reveals a lack of accuracy either in the determined local 3D fibre directions or in the determined geometry of the growth layer surface. Such discrepancy is in the following termed an error (the unit of it being degrees). Figure 11 shows a 3D view of the growth layer within the live knot region, i.e. the layer that is also shown in 

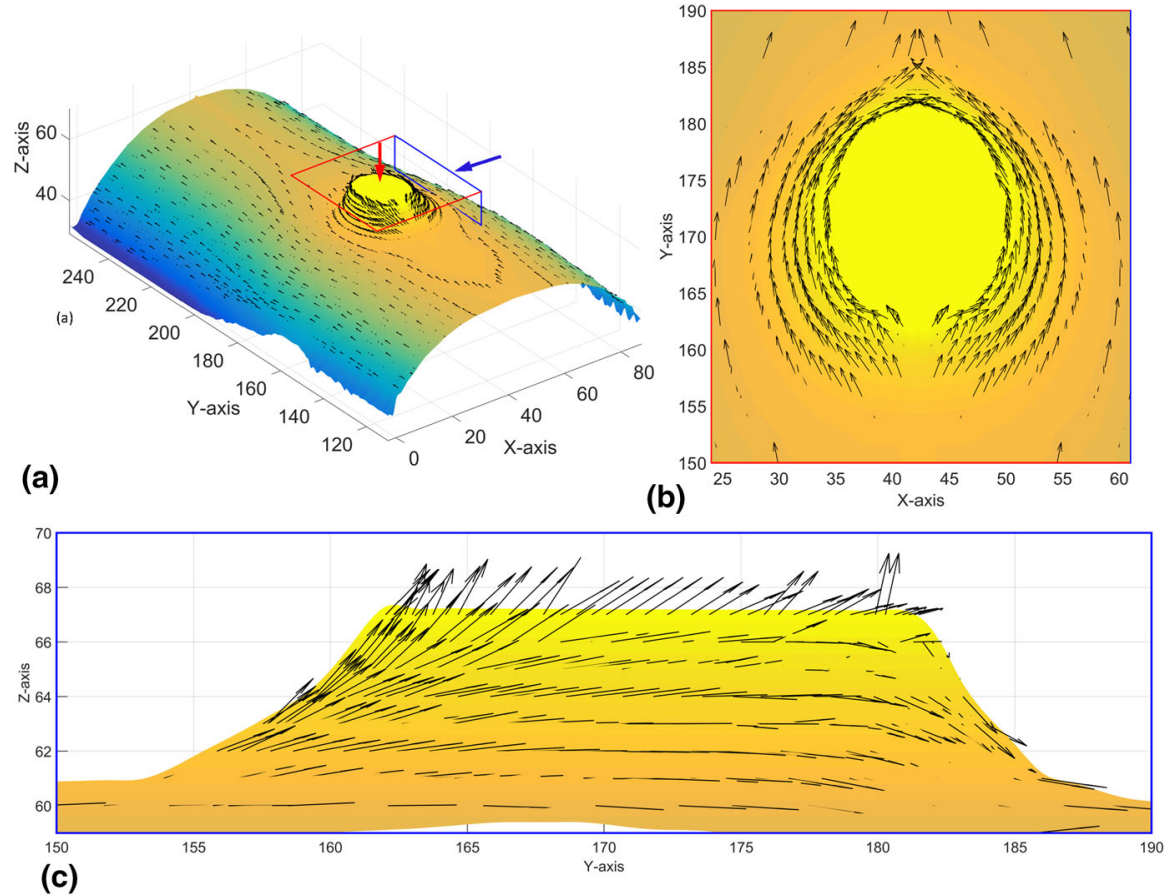

Fig. 10 Results of established 3D fibre directions for the growth layer within the live knot region. A growth layer surface, in which the colour indicates the variation of $z$-coordinate, in the vicinity of a knot is shown together with local fibre directions represented by small black arrows; a 3D view, b partially enlarged $x y$-view and c partially enlarged $y z$-view

Fig. 8. The points displayed represent the manually collected positions that belong to this growth layer surface. In each of these positions, a 3D vector that represented the local fibre direction and a normal vector to the growth layer surface was determined. The colours of the dots indicate the error in the sense that a $90^{\circ}$ angle between the two vectors means no error and any other angle means an error equal to the difference between the determined angle and the $90^{\circ}$ angle. The colour map to the right in Fig. 11 spans from deep blue (no error) to deep red ( $20^{\circ}$ error or more). The colour of the dots indicates that the determined 3D fibre direction vectors and the determined growth layer surface are, in most positions, accurate, errors being in general below $5^{\circ}-6^{\circ}$. However, in some positions, the errors are larger especially in an area just below the knot, but the errors are smaller than $15^{\circ}$, except for the outmost ring encircling the knot, i.e. the data points at the largest $z$-coordinate displayed. This method for assessment of accuracy is not able to distinguish how much of the error relates to the determined 3D fibre directions and how much of that relates to the geometry of the determined growth layer surface, but it proves that none of these error sources causes very large errors. 


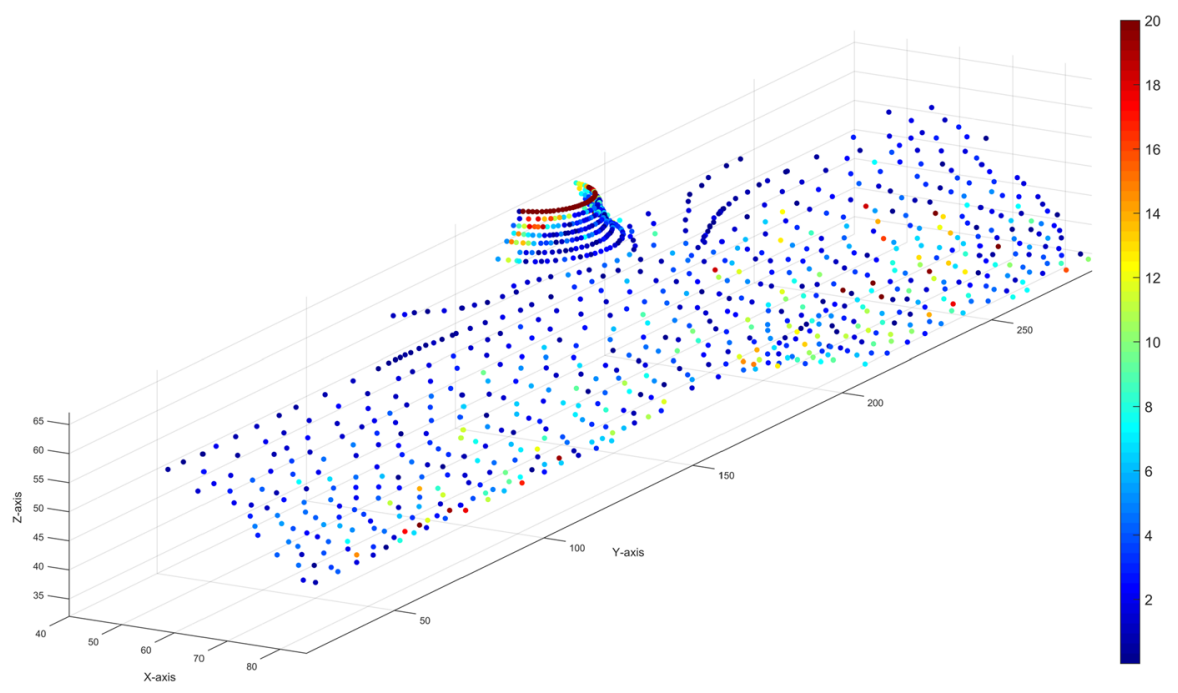

Fig. 11 3D view where dots indicate positions on a growth layer surface (the one within the live knot region, also shown in Figs. 8, 10). In each position, a 3D vector that represents the local fibre direction, a normal vector to the growth layer surface and the angle between these two vectors were determined. The difference between this angle and a $90^{\circ}$ angle constitutes an error (the unit of it being degrees) here represented by the colour of the dots. The colour map to the right spans between deep blue (no error) to deep red $\left(20^{\circ}\right.$ error or more)

\section{Fibre flow lines on growth surfaces}

Figure 12 shows parts of the same growth layer surfaces as those shown in Fig. 8, i.e. one growth layer in the live knot region, displayed by blue colour, and one growth layer within the dead knot region, displayed by yellow colour. Figure 12 shows (a) a 3D view, (b, c) $x y$-views and (d) $y z$-views. Added red and black lines in Fig. 11 represent fibre directions, or fibre flow lines, in the planes of the growth layer surfaces. The red and black lines were established as follows. Starting at an arbitrary position on a growth layer surface, the local 3D fibre direction in that position was determined. Then, a short step, an increment of $1 \mathrm{~mm}$, was taken in the designated direction, giving a new position, i.e. a new xyz-coordinate. This new position was in general not located exactly on the growth layer surface. However, by projecting this position onto the growth layer surface, i.e. by identifying the closest point on the surface, a new position on the surface was obtained. Here, a new 3D fibre direction was determined and a new short step, an increment of $1 \mathrm{~mm}$, was taken in the new designated direction, and so forth. This was repeated until the limit of the data volume, i.e. any limit of $x$-, $y$ - or $z$-coordinate of the scanned volume or the growth layer surface was reached. Each red or black line in Fig. 12 was thus formed by connecting all the positions, originating from a particular start position, on the growth layer surface. The start position for a line is marked with a dot in the same colour as the line. Red colour is used for lines with starting positions below the knot, i.e. at low $y$-coordinates, whereas black colour is used for lines with starting 

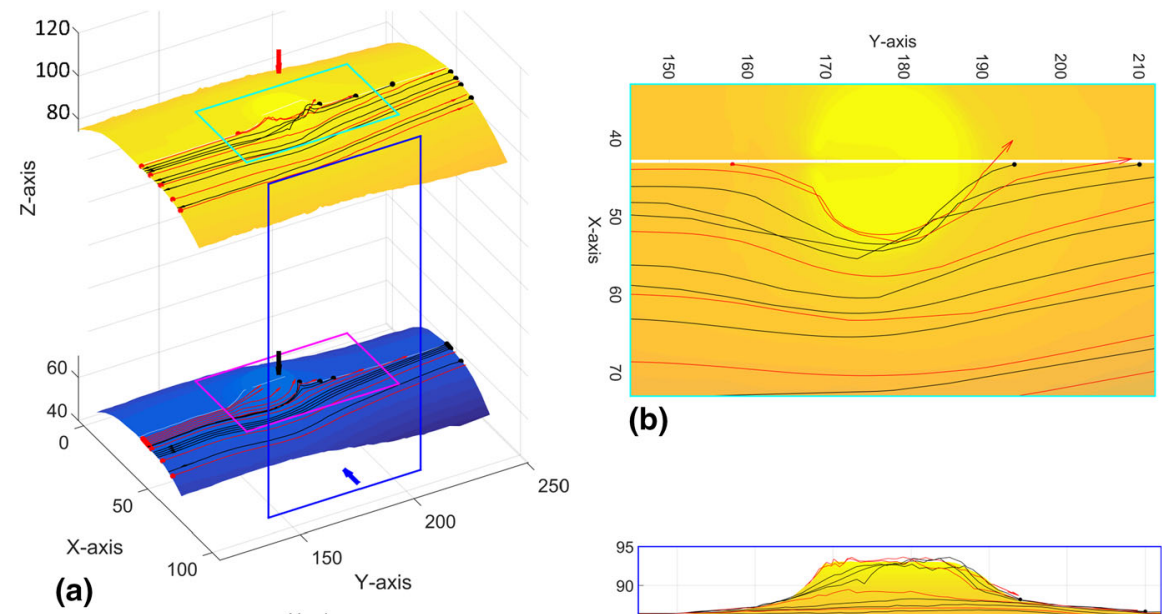

(b)

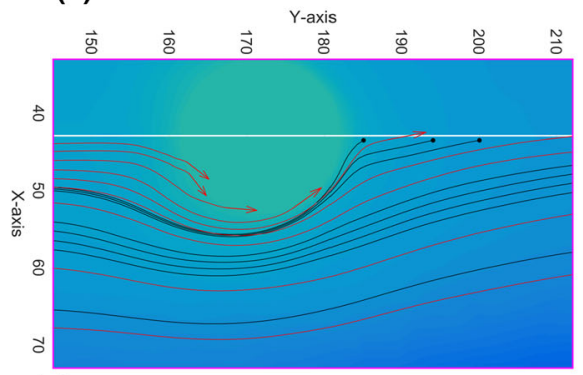

(c)

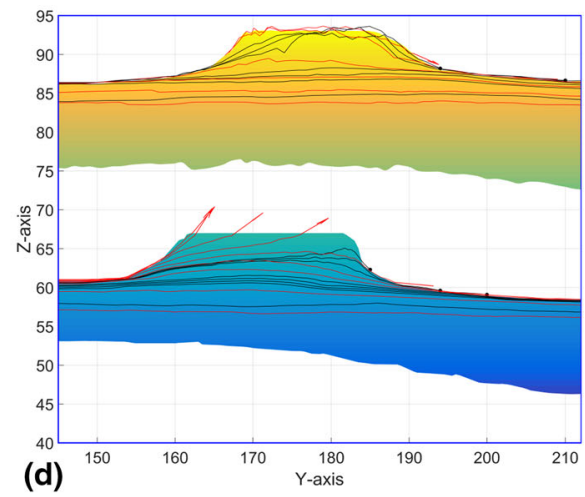

Fig. 12 Fibre flow lines drawn on two different growth layer surfaces. The blue coloured surface is within the live knot region, and the one shown with yellow colour is within the dead knot region (same surfaces as shown in Fig. 8). Red lines represent fibre flow lines with starting positions below the knot (i.e. at a low $y$-coordinate) and then flowing in direction upward towards the top of the tree, whereas black lines represent fibre flow lines with starting positions above the knot (i.e. at a high $y$-coordinate) and flowing downwards; a A 3D view, b, c partially enlarged $x y$-views and $\mathbf{d}$ partially enlarged $y z$-views

positions above the knot. At the end of the lines, arrows pointing in the determined fibre direction at the last position assessed on the growth layer surfaces are drawn.

For the blue surface in Fig. 12, which is within the live knot region, the red fibre flow lines show that the trunk fibres integrate with the branch from below if the starting position is located below the knot, close enough to the assumed plane of symmetry of the specimen. A fibre flow line starting immediately below the knot, very close to the assumed plane of symmetry, integrates with the lower part of the branch, whereas a fibre flow line that starts below the knot, but a little bit to the side of the assumed plane of symmetry, integrates with the intermediate or even top part of the branch. When the starting position is located below the knot but at a longer distance from the assumed plane of symmetry, the fibre flow line goes smoothly around the knot, i.e. it does not integrate with the branch at all. Moreover, when the starting position of a fibre flow line is located above the knot (this is the case for all the lines drawn with black colour) lines always go around the branch and do not 
integrate with the branch. Note that this is the case even when the start position is located immediately above the knot and very close to the assumed plane of symmetry, which is clearly shown in Fig. 12c. Thus, the results displayed for the live knot region are, in this respect, in agreement with the hypothesis of Shigo (1985). The results give no support to his hypothesis of an altering fibre pattern over the growth season. However, any such alteration (within a fraction of an annular ring) would be on a scale beyond reach for this investigation giving a resolution of no more than one to two millimetres.

The fibre flow lines drawn on the yellow surface, which is within the dead knot region, go around the knot and do not integrate with the branch. This is the case no matter if the starting position of the fibre flow line is located below (red lines) or above (black lines) the knot. Of course, it must be this way since the very definition of a dead knot is that it no longer grows and integrates with the trunk. The results displayed give clear indications, however, that the method developed to determine $3 \mathrm{D}$ fibre orientation is capable of representing the 3D fibre orientation accurately on a detailed level in the close surroundings of knots. Regarding the appearance of the fibre flow lines in different regions, it may be added that the determined lines in the live knot region (blue surface) are smooth/regular in shape, whereas the fibre flow lines close to the knot in the dead knot region (yellow surface) are more irregular. The latter is due to the fact that there is a thin layer of bark here, between the knot tissue and the trunk tissue, and thus, locally, no fibre orientation to determine using the tracheid effect.

\section{Conclusion}

A laboratory method to assess the geometry of growth layers and the 3D directions of fibres within wood specimens containing knots was presented. The method is based on optical flatbed scanning and tracheid effect scanning, giving surface images and in-plane fibre directions in high-resolution grids, respectively. The growth layer geometry was established using the surface images. By assuming that a plane of symmetry exists through a knot, splitting a specimen including a knot through this plane of symmetry into two parts and examining the fibre orientation in planes orthogonal to each other, the $3 \mathrm{D}$ fibre orientation in the surroundings of the knot was determined.

The laboratory method allows for quantitative assessment of 3D fibre directions in any position close to a knot. Regarding the obtained accuracy, it was shown that the angle between fibre direction and the normal direction of a growth layer surface was, when both directions were determined at the same position, in general very close to $90^{\circ}$. Since fibres must be oriented within the plane of a growth layer, any discrepancy from a $90^{\circ}$ angle would suggest an error. The discrepancy was used for the assessment of accuracy indicating that the errors of determined local fibre directions were small, in general smaller than $5^{\circ}-6^{\circ}$.

The obtained results of growth layers show that a knot/branch causes a pronounced local effect, a swelling, on the geometry of the growth layer close to the knot, whereas the bulk of the growth layer forms a cylindrically shaped surface with 
its longitudinal direction close to parallel with the pith of the tree/log. Moreover, it was shown that fibre flow lines visualized on growth layer surfaces integrate the branch, in live knot regions, with the stem from below. On the other hand, if a starting position for a fibre flow line is set immediately above a branch, and followed downward, the fibre flow line does not integrate with the knot but pass around it, downward along the stem. Thus, the results confirm what has been assumed by Shigo (1985) and others that only trunk tissue from below the branch connects with the branch. The results give no support to Shigos hypothesis of an altering fibre pattern over the growth season. However, any such alteration (within a fraction of an annular ring) would be on a scale beyond reach for this investigation giving a resolution of no more than one to two millimetres.

Further work should comprise both refinement and utilization of the developed method. Regarding refinement, spiral grain, being in itself anti-symmetric rather than symmetric with respect to the plane through the centre of the knot, may be considered. This can be done since detected fibre directions on longitudinaltangential planes in clear wood areas give knowledge of spiral grain as a function of the radial distance to the pith for the investigated specimen. Regarding utilization, the quantitative data obtained using the method should be used to calibrate parameters of mathematical models of growth layer geometry and fibre orientation close to knots in wood, such as the one suggested by Foley (2003). Since local fibre orientation in timber is decisive for strength, knowledge gained on the basis of results from the method presented should also be utilized in future methods for accurate prediction of timber strength.

Open Access This article is distributed under the terms of the Creative Commons Attribution 4.0 International License (http://creativecommons.org/licenses/by/4.0/), which permits unrestricted use, distribution, and reproduction in any medium, provided you give appropriate credit to the original author(s) and the source, provide a link to the Creative Commons license, and indicate if changes were made.

\section{References}

Briggert A, Hu M, Olsson A, Oscarsson J (2016) Evaluation of three dimensional fibre orientation in Norway spruce using a laboratory laser scanner. In: World conference on timber engineering, August 22-25, Vienna, Austria

Dodoo A, Gustavsson L, Sathre R (2009) Carbon implications of end-of-life management of building materials. Resour Conserv Recycl 53:276-286

Dodoo A, Gustavsson L, Sathre R (2012) Effect of thermal mass on life cycle primary energy balances of a concrete- and a wood-frame building. Appl Energy 92:462-472

Foley C (2001) A three-dimensional paradigm of fibre orientation in timber. Wood Sci Technol $35: 453-465$

Foley C (2003) Modelling the effect of knots in structural timber. Dissertation, Lund University

Goodman JR, Bodig J (1978) Mathematical model of the tension behavior of wood with knots and cross grain. In: Proceedings from the first international conference on wood fracture, August 14-16, Banff, Alberta

Goodman JR, Bodig J (1980) Tension behavior of wood-an anisotropic, inhomogeneous material. Structural research report no. 32. Colorado State University, Fort Collins

Hackspiel C (2010) A numerical simulation tool for wood grading. Dissertation, Vienna University of Technology

Harris JM (1988) Spiral grain and wave phenomena in wood formation. Springer, Berlin 
Hu M, Briggert A, Olsson A, Johansson M, Oscarsson J, Säll H (2016) Three dimensional growth layer geometry and fibre orientation around knots-a laboratory investigation. In: World conference on timber engineering, August 22-25, Vienna, Austria

Lang R, Kaliske M (2013) Description of inhomogeneities in wooden structures: modelling of branches. Wood Sci Technol 47:1051-1070

Lukacevic M, Füssl J (2014) Numerical simulation tool for wooden boards with a physically based approach to identify structural failure. Eur J Wood Prod 72:497-508

Mattheck C (1997) Design in nature: learning from trees. Springer, Berlin

Matthews PC, Beech BH (1976) Method and apparatus for detecting timber defects. U.S. patent no. $3676384 \mathrm{~A}$

Nelly D (1991) Water transport at stem-branch junctures in woody angiosperms. J Arboric 17(11):285-290

Olsson A, Oscarsson J (2017) Strength grading on the basis of high resolution laser scanning and dynamic excitation: a full scale investigation of performance. Eur J Wood Prod 75(1):17-31

Olsson A, Oscarsson J, Serrano E, Källsner B, Johansson M, Enquist B (2013) Prediction of timber bending strength and in-member cross-sectional stiffness variation on the basis of local wood fibre orientation. Eur J Wood Prod 71(3):319-333

Panshin AJ, Zeeuw C (1980) Textbook of wood technology, 4th edn. McGraw-Hill Book Company, New York

Plomion C, Leprovost G, Stokes A (2001) Wood Formation in Trees. Plant Physiol 127:1513-1523

Säll H (2002) Spiral grain in Norway spruce. Dissertation, Växjö University

Shigo AL (1985) How tree branches are attached to trunks. Can J Bot 63(8):1391-1401

Shigo AL (1986) A new tree biology: facts, photos, and philosophies on trees and their problems and proper care. Shigo and Trees, Associates, Durham

Slater D, Harbinson C (2010) Towards a new model of branch attachment. Arboric J 33:95-105

Soest J, Matthews PC, Wilson B (1993) A simple optical scanner for grain defects. In: Proceedings of 5th international conference on scanning technology and process control for the wood products industry, October 25-27, Atlanta, USA 